\title{
Fluctuations and the Energy-Optimal Control of Chaos
}

\author{
I. A. Khovanov, ${ }^{1}$ D. G. Luchinsky, ${ }^{2}$ R. Mannella, ${ }^{3}$ and P. V. E. McClintock ${ }^{2}$ \\ ${ }^{1}$ Department of Physics, Saratov State University, Astrahanskaya 83, 410026, Saratov, Russia \\ ${ }^{2}$ Department of Physics, Lancaster University, Lancaster, LAl 4YB, United Kingdom \\ ${ }^{3}$ Dipartimento di Fisica, Università di Pisa and INFM UdR Pisa, Piazza Torricelli 2, 56100 Pisa, Italy
}

(Received 28 March 2000)

\begin{abstract}
The energy-optimal entraining of the dynamics of a periodically driven oscillator, moving it from a chaotic attractor to a coexisting stable limit cycle, is investigated via analysis of fluctuational transitions between the two states. The deterministic optimal control function is identified with the corresponding optimal fluctuational force, which is found by numerical and analog simulations.
\end{abstract}

PACS numbers: 05.45.Gg, 02.50.-r, 05.20.-y, 05.40.-a

The stability of chaotic systems in the presence of noise and methods for controlling these systems are of intrinsic interdisciplinary interest and of obvious importance in relation to applications. Methods already available [1] for the control of chaos include entraining to a chosen "goal dynamics," which necessarily requires large modifications of the system's dynamics [2,3], and a variety of minimal forms of interaction [4-6] which have hitherto been restricted by the linear approximations adopted.

The energy-optimal implementation of deterministic switching from the basin of attraction of a chaotic attractor (CA) has remained an unsolved archetypal problem [7] for a long time. Its solution must amount to an important extension of the range of model-exploration objectives (cf. [2] and [5]) achievable through minimal control techniques. At the same time, the seemingly separate question of noise-induced escape from the basin of attraction of a $\mathrm{CA}$ has remained a major scientific challenge ever since the first attempts to generalize the classical escape problem to cover this case [8]. These two apparently quite different problems are usually considered separately within the distinct subfields of deterministic and stochastic nonlinear dynamics. See, however, Ref. [9] for a discussion of the interrelationship between stochastic and control problems, and, in particular, [9](c) for the analogy between their variational formulations.

In this Letter we show how the energy-optimal control of chaos can be effected via an analogy between the variational formulations of both problems using a statistical analysis of fluctuational trajectories. The main difficulty in tackling these problems stems from the complexity of the system dynamics near a CA and is related, in particular, to delicate questions concerning the uniqueness of the solution and the boundary conditions at a CA. The approach proposed below is based on the analysis of an oscillator interacting with a thermal bath. In the zero-noise intensity limit, a consistent theoretical development $[10,11]$ from the microscopic to the macroscopic equations of motion leads to descriptions of both its deterministic (dissipative) and fluctuational dynamics within the framework of Hamiltonian formalism [12]. It can be shown both on physical grounds and rigorously that the Wentzel-Freidlin Hamiltonian [12] arising in this approach is equivalent to the Pontryagin Hamiltonian in the control problem [7] with an additive linear unrestricted control; the corresponding optimal control function is equivalent to the optimal fluctuational force [9](c).

We illustrate the approach by analyzing the motion of a periodically driven nonlinear oscillator

$$
\begin{aligned}
\dot{q}_{1} & =K_{1}(\mathbf{q}(t))=q_{2}, \\
\dot{q}_{2} & =K_{2}(\mathbf{q}(t))+u(t) \\
& =-2 \Gamma q_{2}-\omega_{0}^{2} q_{1}-\beta q_{1}^{2}-\gamma q_{1}^{3}-h \cos (\Omega t)+u(t) .
\end{aligned}
$$

Here $u(t)$ is the control function. Parameters were chosen such that the potential is monostable $\left(\beta^{2}<4 \gamma \omega_{0}^{2}\right)$, the dependence of the energy of oscillations on their frequency is nonmonotonic $\left(\frac{\beta^{2}}{\gamma \omega_{0}^{2}}>\frac{9}{10}\right)$, and the motion is underdamped $\Gamma \ll \Omega \approx 2 \omega_{0}$. This model is of interest in a number of contexts in which theoretical analysis is possible for a wide range of parameter values [13]. It is a system in which chaos can be observed at relatively small values $h \approx 0.1$ of the driving force amplitude.

For a given damping $(\Gamma=0.025)$ the amplitude and frequency of the driving force were chosen so that the chaotic attractor coexists with the stable limit cycle (SC in Fig. 1). The chaotic state appears via period-doubling bifurcations and thus corresponds to a nonhyperbolic attractor (NHA). Its boundary of attraction $\partial \Omega$ is nonfractal and is formed by the saddle cycle of period 1 (S1). For details about the phase diagram, see Ref. [14].

We have considered the following energy-optimal control problem. The system (1) with unconstrained control function $u(t)$ is to be steered from the NHA to the stable limit cycle in such a way that the energy ("cost") functional $R$ is minimized, with $t_{1}$ unspecified,

$$
R=\inf _{u \in U} \frac{1}{2} \int_{t_{0}}^{t_{1}} u^{2}(t) d t .
$$

Here the control set $U$ consists of functions (control signals) which are able to move the system from the NHA to the SC. 


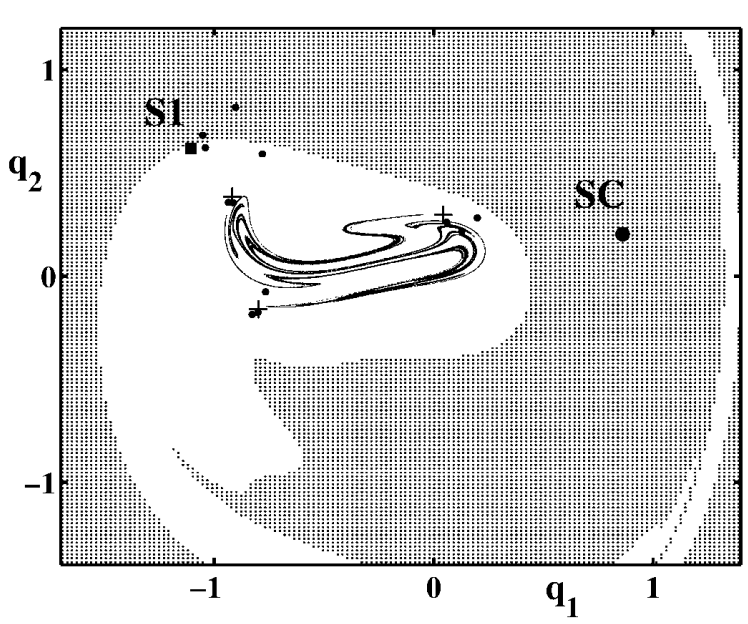

FIG. 1. The basins of attraction of the stable limit cycle SC (shaded) and NHA (white) for a Poincaré cross section with $\Omega t=0.6 \pi(\bmod 2 \pi), \Omega=0.95$. The largest Lyapunov exponent for the NHA is 0.0449 . The period- 1 saddle cycle $\mathrm{S} 1$ at the NHA boundary $\partial \Omega$ is shown by the filled square. The saddle cycle of period 3 is shown by plusses. Intersections of the actual escape trajectory with the Poincare cross section are indicated by the small filled circles.

It can be shown (see, e.g., Ref. [7]) that, if a solution of the control problem $[\bar{u}(t), \mathbf{q}(t)]$ exists, there also exists a continuous piecewise differentiable function $\mathbf{p}(t)=$ $\left\{p_{1}(t), p_{2}(t)\right\}$ such that

$$
\begin{gathered}
\dot{q}_{i}=\frac{\partial H_{c}}{\partial p_{i}}, \quad \dot{p}_{i}=-\frac{\partial H_{c}}{\partial q_{i}}, \quad i=\{1,2\}, \\
H_{c}=1 / 2 p_{2}^{2}+p_{1} K_{1}+p_{2} K_{2} .
\end{gathered}
$$

Here the variables $p_{1}(t), p_{2}(t)$ are not simultaneously zero and it is assumed that the optimal control function $\bar{u}(t)$ at each instant takes those values $u(t)=p_{2}$ that maximize $H_{c}$ over $U$.

The function $R$ in (2) coincides (up to a constant scaling factor) with the definition of the activation energy for fluctuational transitions between the NHA and the SC if the control signal $u(t)$ in (1) is substituted with zero-mean white Gaussian noise $\xi(t)[12]$ such that

$$
\langle\xi(t)\rangle=0, \quad\langle\xi(t) \xi(0)\rangle=D \delta(t) .
$$

Correspondingly, the function $H_{c}$ (3) coincides with the Hamiltonian $H$ of an auxiliary Hamiltonian system (3) which determines the optimal fluctuational paths (see, e.g., Refs. $[12,15])$. Thus the optimal control signal $\bar{u}(t)$ can be identified with the optimal fluctuational force [9](c) which drives the system from the NHA to the SC. We note that both $\bar{u}(t)$ and the optimal force are related to $p_{2}$ in (3). This interrelationship is intuitively clear because, in thermal equilibrium $\left(D=4 \Gamma k_{B} T\right)$, the probability of fluctuations is determined by the minimum work from the external source needed to produce the given change in the thermodynamic quantities $\rho \propto \exp \left(-R_{\min } / k_{B} T\right)$ [16]. We therefore suggest that the optimal control function $\bar{u}(t)$ can be found experimentally by measurement of the optimal fluctuational force $[17,18]$.

We have tested this idea through analog electronic modeling [18] of (1) and digital simulation following the prescriptions of [19]. Qualitatively similar results were obtained but, because precision is of particular importance here, the data reported below are those from the digital simulations. The underlying idea is that, when the system (1) is driven by a random force $\xi(t)(4)$ instead of $u(t)$, it will occasionally fluctuate to $\partial \Omega$. In doing so, in the limit where the noise intensity tends to zero, the system will follow very closely the deterministic trajectories of (3).

For the technique to be applicable a solution of (3) moving the system from the NHA to $\partial \Omega$ must exist, and one has to be able to identify the boundary conditions for this solution on the NHA. Earlier numerical simulations [20] demonstrated that a solution does indeed exist and that it is typically unique in the limit $D \rightarrow 0$.

The method involves monitoring the system continuously and collecting all successful realizations $\left[q_{1}^{\text {esc }}(t)\right.$, $\left.q_{2}^{\text {esc }}(t), \xi^{\text {esc }}(t)\right]$, moving it from the NHA to $\partial \Omega$. From these realizations, a time-dependent prehistory distribution is built [17]. In the present case, this distribution turns out to be characterized by a narrow ridge, as the noise intensity is decreased, allowing us to define an approximate solution $\tilde{u}(t)$ for the control function [the exact solution is $\left.\bar{u}(t)=\lim _{D \rightarrow 0} \tilde{u}(t)\right]$, corresponding to the maximum of the distribution. The boundary conditions are found by analysis of how the energy-optimal escape path $\left[\left\langle q_{1}^{\text {esc }}(t)\right\rangle,\left\langle q_{2}^{\text {esc }}(t)\right\rangle\right]$ merges with the NHA. Note that the topological features of the prehistory distribution yield direct insight into the control problem: where the prehistory distribution does not develop a well-defined ridge in the $D \rightarrow 0$ limit, we may infer that control via a simple function is not achievable.

An actual escape trajectory is indicated in Poincaré cross section by the filled circles in Fig. 1. A typical optimal escape path and the corresponding optimal force, obtained by averaging a few hundred such trajectories, are shown in Fig. 2. Analysis of the optimal path reveals that the system leaves the NHA along the unstable manifold of the saddle cycle of period 5 (S5 with multipliers $\mu_{1}=$ $0.04157<1$ and $\mu_{2}=4.60403728>1$ ) embedded in the NHA. At this moment the optimal fluctuational force $\left\langle\xi^{\text {esc }}(t)\right\rangle$ switches on, driving the system to $\partial \Omega$ via the saddle cycle of period 3 (S3 with multipliers $\mu_{1}=$ $0.04873<1$ and $\mu_{2}=7.608312>1$ ). Near the saddle cycle S1 that forms the boundary of the basin of attraction the optimal force dies out. Note that no action is required to bring the system from $\mathrm{S} 1$ to the stable limit cycle. As discussed above, this path is an approximation (because of the finite intensity of the noise) of the optimal control function $\bar{u}(t)$.

It can be seen from Fig. 1 that the saddle cycle $S 3$ is not embedded in the NHA. It is probably the nearest saddle 


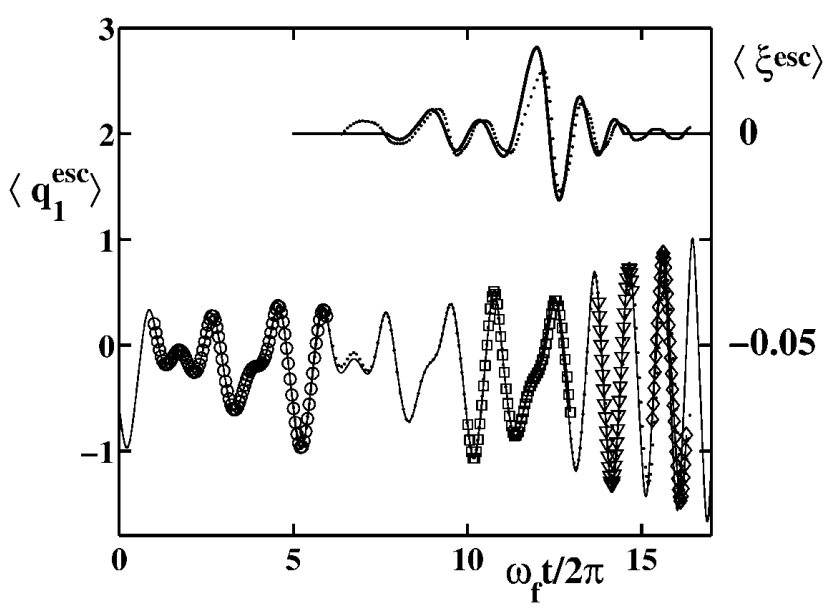

FIG. 2. The most probable escape path (bottom solid curve) from the NHA to SC, found in numerical simulations with $h=$ $0.13, \omega_{f} \approx 0.95, \omega_{0} \approx 0.597$, and $T \approx 0.0005$. Single periods of the saddle cycles of period 5, 3, and 1 are shown by open circles, squares, and triangles, respectively; the stable limit cycle is shown by diamonds. Top curve: the corresponding optimal force after filtration. The optimal path and optimal fluctuational force found by the solution of the boundary value problem are shown in each case as dotted lines.

cycle to the boundary of the basin of attraction of the NHA in terms of the action variable, and can be considered as the boundary of the NHA itself.

Thus we conclude that the solution $\tilde{u}(t)$ and the corresponding boundary conditions can be found using our new experimental method. Moreover the escape problem has in this case been reduced to the analysis of transitions between three saddle cycles $\mathrm{S} 5 \rightarrow \mathrm{S} 3 \rightarrow \mathrm{S} 1$, in qualitative agreement with the well known statement that saddle cycles provide detailed invariant characterizations for dynamical systems of low intrinsic dimension (see, e.g., $[21,22])$. We note that the solution found is independent of the initial conditions on the chaotic attractor: the transient time required for the system to reach S5 (in the presence of noise) from arbitrary initial conditions is exponentially smaller then average escape time, and the quasiperiodic steady state distribution is formed on the attractor prior to escape [23].

Once boundary conditions are specified one can solve the corresponding boundary value problem for the system (3) numerically. The results of the numerical solution of the boundary value problem obtained by the relaxational method are shown in Fig. 2 by the dotted lines and are in good agreement with the solution found from the analysis of fluctuational trajectories.

To verify that the optimal force $\tilde{u}(t)$ found in the experiment really does minimise the energy of the control function steering the system (1) from the NHA to the SC1, we set it to arbitrary initial conditions in the basin of attraction of the NHA and let it evolve deterministically until it passed through the initial part of the unstable manifold of S5. At that moment the deterministic control function was switched on. For a given shape of the control function and/ or initial conditions, its amplitude was set to the threshold for switching of the system from chaotic to regular motion on SC1.

It was found that the system is very sensitive to small variations in the control function: any deviation from the shape of $\tilde{u}(t)$, or from the initial conditions found in the experiment, leads to a substantial increase in the energy required to attain $\mathrm{SC} 1$. Some experimental results are shown in Fig. 3. It can be seen that the energy of the control function is approximately twice as large if the optimal force is approximated by the sin function modulated by a Gaussian $u(t)=a_{1} \sin \left(a_{2} t\right) \exp \left[-\left(t-a_{3}\right)^{2} a_{4}\right]$, and it is, respectively, $\sim 4$ and $\sim 20$ times larger if the optimal force is approximated by rectangular pulses or distorted with an arbitrary low-frequency perturbation.

We have also performed experiments using an open-plusclosed loop control technique [2] and an adaptive control algorithm [3] to steer the system from the NHA to the
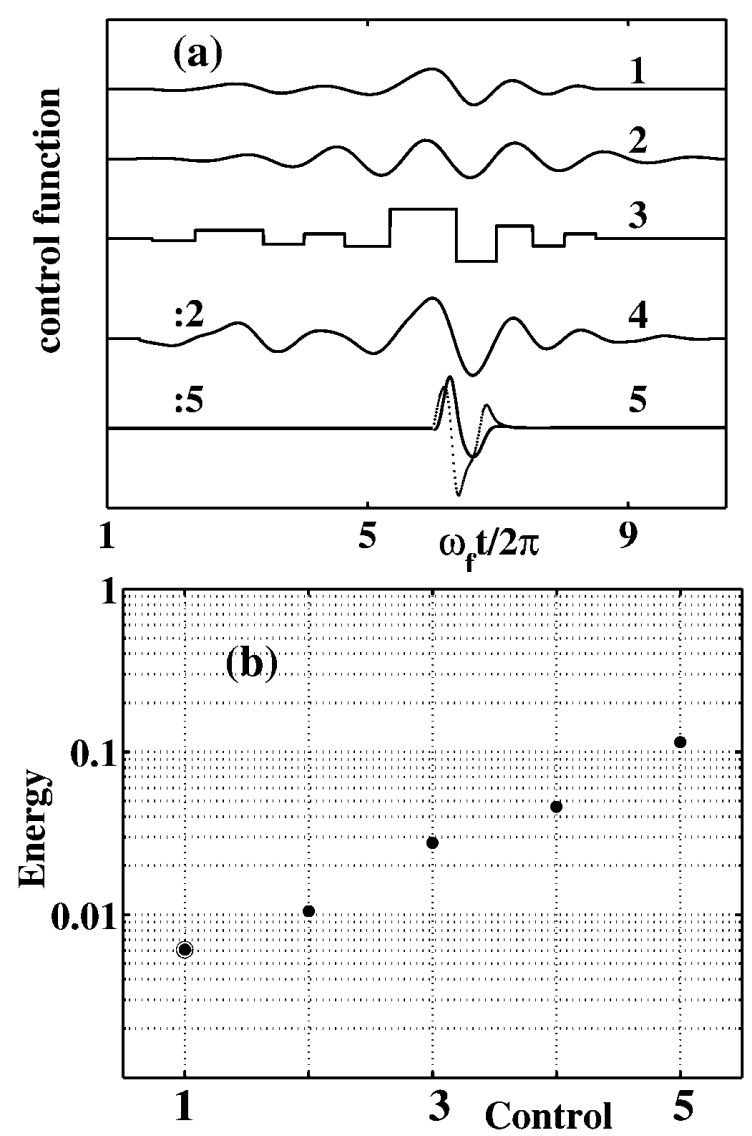

FIG. 3. (a) Threshold control functions (note differing vertical scales) inducing switching in the numerical experiment: 1: optimal force found by statistical analysis of fluctuational escape trajectories; 2: its approximation by $u(t)=a_{1} \sin \left(a_{2} t\right) \times$ $\exp \left[-\left(t-a_{3}\right)^{2} a_{4}\right]$, where $a_{i}$ are constants; 3: approximation by rectangular pulses; 4 : arbitrary distortion of the optimal force by a low-frequency perturbation; 5: control functions produced in open-plus-closed loop control [2] for $q_{1}$ (solid line) and $q_{2}$ (dotted line). (b) Energies of the control functions shown in (a). 
SC1. Although these methods are designed to optimize the recovery time, rather than to minimize the energy of the control function, they are efficient in entraining the system dynamics to the goal dynamics. So it is interesting to compare their performance with that of the control function found in our experiment. The energy of the control functions (see Fig. 3) obtained by these methods is more than an order of magnitude larger then the energy of the optimal control function $\tilde{u}(t)$ found by our new technique. The best results obtained by the open-plus-closed loop control are also shown in Fig. 3. Full details, together with the numerical solution of the boundary value problem, will be given elsewhere [24].

Of course, the time required for the system to approach S5 (which is where the optimal control force can be switched on) varies for different initial conditions on the NHA: it is typically $\epsilon^{D_{p}}$, where $\epsilon$ is the linear dimension of the region and the $D_{p}$ is the pointwise dimension of a periodic point in this region [22]. In order to reduce this initial waiting period, and thus the average transition time, one could apply the techniques [6,25] developed earlier for effecting switching between controlled saddle periodic orbits embedded in an NHA.

In conclusion, we have proposed a novel technique for the energy-optimal steering of a nonlinear oscillator away from the basin of attraction of an NHA, and we have verified experimentally that it works in a particular case. The technique can readily be combined with established minimal forms of control, extending substantially the range of model-exploration objectives that can be achieved by such methods. Preliminary investigations [14] indicate that the technique may also be applied in the case of the Lorenz attractor, whose ergodic properties are very different, and modifications are needed to take account of the finite transition time. We infer that it can be further extended to treat cases where the boundaries of attraction are fractal. The possibility of applying the technique to higher dimensional systems is an open problem of immediate interest.

The research was supported by INTAS (Grants No. N 96-0305, No. N YSF 99-3920), by the Royal Society of London, and by the Engineering \& Physical Sciences Research Council (UK).

[1] A. L. Fradkov and A. Y. Pogromsky, Introduction to Control of Oscillations and Chaos, Series on Nonlinear Science A, Vol. 35 (World Scientific, Singapore, 1998).

[2] E. A. Jackson, Chaos 7, 550 (1997).

[3] S. P. Raj and S. Rajasekar, Phys. Rev. E 55, 6237 (1997).
[4] T. Shinbrot, C. Grebogi, J. Ott, and E. Yorke, Nature (London) 363, 411 (1993); D. Auerbach, C. Grebogi, E. Ott, and J. A. Yorke, Phys. Rev. Lett. 69, 3479 (1992); D. Xu and S. Bishop, Chaos Solitons Fractals 4, 1931 (1994); S. R. Bishop and D. Xu, Phys. Rev. E 54, 3204 (1996).

[5] B. Hübinger, R. Doerner, W. Martienssen, M. Herdering, R. Pitka, and U. Dressler, Phys. Rev. E 50, 932 (1994).

[6] E. Barreto et al., Phys. Rev. E 51, 4169 (1995).

[7] L. C. Young, Lectures on the Calculus of Variations and Optimal Control Theory (Saunders, Philadelphia, 1969); P. Hagedorn, Non-linear Oscillations (Clarendon Press, Oxford, 1982).

[8] R. Kautz, Phys. Lett. A 125, 315 (1987); P. Grassberger, J. Phys. A 22, 3283 (1989); R. Graham, A. Hamm, and T. Tel, Phys. Rev. Lett. 66, 3089 (1991).

[9] (a) W. H. Fleming, Appl. Math. Optim. 4, 329 (1978); (b) P. Whittle, Risk-Sensitive Optimal Control (Wiley, Chichester, 1990); (c) V. N. Smelyanskiy and M. I. Dykman, Phys. Rev. E 55, 2516 (1997).

[10] R. Zwanzig, J. Stat. Phys. 9, 215 (1973).

[11] M. I. Dykman and M. Krivoglaz, in Soviet Physics Reviews, edited by I. M. Khalatnikov (Harwood Academic, New York, 1984), Vol. 5, pp. 265-442.

[12] M. I. Freidlin and A. D. Wentzel, Random Perturbations in Dynamical Systems (Springer, New York, 1984).

[13] S. M. Soskin et al., Int. J. Bifurcation Chaos 7, 923 (1997); R. Mannella, S. M. Soskin, and P. V. E. McClintock, Int. J. Bifurcation Chaos 8, 701 (1998).

[14] I. A. Khovanov et al., in Stochastic and Chaotic Dynamics in the Lakes, edited by D. S. Broomhead et al. (AIP, Melville, NY, 2000), pp. 48-53.

[15] R. S. Maier and D. L. Stein, SIAM J. Appl. Math. 57, 752 (1997); V. N. Smelyanskiy, M. I. Dykman, and R. S. Maier, Phys. Rev. E 55, 2369 (1997).

[16] L. D. Landau and E. M. Lifshitz, Statistical Physics (Pergamon, New York, 1980), 3rd ed., Part 1.

[17] D. G. Luchinsky, J. Phys. A 30, L577 (1997); D. G. Luchinsky and P. V.E. McClintock, Nature (London) 389, 463 (1997).

[18] D. G. Luchinsky, P. V.E. McClintock, and M. I. Dykman, Rep. Prog. Phys. 61, 889 (1998).

[19] R. Mannella, in Supercomputation in Nonlinear and Disordered Systems, edited by L. Vázquez, F. Tirando, and I. Martin (World Scientific, Singapore, 1997), pp. 100-130.

[20] D. G. Luchinsky and I. A. Khovanov, JETP Lett. 69, 825 (1999).

[21] D. Auerbach et al., Phys. Rev. Lett. 58, 2387 (1987); P. Schmelcher and F. K. Diakonos, Phys. Rev. Lett. 78, 4733 (1997).

[22] C. Grebogi, E. Ott, and J. A. Yorke, Phys. Rev. A 37, 1711 (1988).

[23] This point will be discussed in detail elsewhere.

[24] I. A. Khovanov et al. (to be published).

[25] D. Xu and S. Bishop, Phys. Rev. E 54, 6940 (1996). 